\title{
COMPLEX PADÉ APPROXIMANTS FOR BIDIRECTIONAL AND NONPARAXIAL BEAM PROPAGATION METHOD
}

\author{
R. Petruškevičius \\ Institute of Physics, Savanoriu 231, LT-02300 Vilnius, Lithuania \\ E-mail: raimisp@ktl.mii.lt
}

Received 31 January 2005

\begin{abstract}
The nonparaxial and bidirectional beam propagation method suitable for modelling near-field and high numerical aperture (NA) optical storage systems is suggested for 2D geometry and TE polarization of incident light beam. The complex Padé approximants are introduced for correct approximation of evanescent field in the near-field optics. Pole-zero shifting and branch-cut rotation methods of building complex Padé approximants are studied and compared.

Keywords: near-field optical storage, bidirectional beam propagation method, nonparaxial beam propagation, evanescent waves, complex Padé approximants
\end{abstract}

PACS: 42.25.Bs, 42.40.Ht, 42.79.Vb

\section{Introduction}

High density optical data storage systems with the capacity of more than a few tens of gigabytes have been attractive for high density TV or digital movies. However, in all optical recording systems a laser beam spot size is limited by diffraction limit depending on the wavelength $(\lambda)$ and numerical aperture (NA) of lens. In order to increase the storage density up to hundreds of gigabytes, optical near-field recording using probes of the scanning near-field microscope has been proposed by Betzing at al. [1]. The development of near-field techniques in optical storage systems promises significant increase in the storage density by using evanescent waves to reduce the size of the optical spot interacting with recordable medium. Several approaches based on either near-field aperture (scanned probe [2], very small aperture laser [3,4]) or solid immersion lens (SIL) $[5,6]$ schemes have been introduced. These methods, however, face challenges of extremely low throughput or need for maintaining small $(\sim 100 \mathrm{~nm})$ and stable recording head-media separation. Super-resolution near-field structures (superRENS) using $\mathrm{Sb}$ [7] or $\mathrm{AgO}_{x}$ [8,9] thin film nonlinear mask layers and integrated near-field optical (INFO) media [10], with nano-optics embedded in the optical medium, were proposed. Such super-RENS and INFO media approaches do not require to maintain small head-media separation. These structures are suitable ways for development of the high density removable optical discs and compatible with future use of blue and UV laser sources for optical recording.

INFO media employ embedded nano-cylinder optics directly in the optical disc [10]. These nano-cylinders have high refraction index (in the case of using $\mathrm{TiO}_{2}$ $n=2.4$ ) and work like micro-SILs with high NA. A large amount of incident light beam undergoes the total internal reflection in the nano-cylinder SILs, and superresolution is achieved by presence of evanescent waves in the formation of beam spot overcoming the diffraction limit under nano-SIL surface. This INFO media approach is also very promising for use together with near-field microholographic recording to increase storage density of the holographic optical disc. To investigate this type of near-field optical storage systems the numerical methods should support wide-angle (tightly focused), nonparaxial beam propagation taking into account evanescent waves. Furthermore, the bidirectional propagation is very important because usually an optical disc produces strong back-reflected beam.

Today, the most popular method for modelling nonparaxial and bidirectional beam propagation, which is proper for simulation of near-field optical storage system, is the finite-difference time-domain (FDTD) method [11] based on direct solution of Maxwell's equations using cells method by Yee [12] together with an explicit finite-difference numerical scheme. Wideangle and bidirectional beam propagation in the FDTD 
method is correctly modelled due to time-dependent simulations. In fact, it is a very numerically expensive method, because for the $3 \mathrm{D}$ geometry we need to solve $4 \mathrm{D}$ problem and this requires long computation times and large memory for storage. To speed up the simulation it is important to remove the time variable from the problem. This fast time-dependent behaviour can be omitted for the optical data storage systems, and is enough to solve the electromagnetic problem in quasistationary limit. The most popular method without time variable is so-called Beam Propagation Method (BPM) [13-15] widely used in integrated optics. The BPM uses solution of Helmholtz wave equation in the paraxial limit of slowly varying envelope and implicit Crank-Nicholson numerical scheme. A lot of BPM realizations were suggested. One of the first realizations was suggested by Feit and Fleck based on the fast Fourier transform (FFT-BPM) [16]. Then Dagli suggested a more efficient method based on finite-difference schemes (FD-BPM) [17]. There are various modifications of BPM for 2D and 3D geometries, TE and TM polarization of incident light, scalar, semi-vectorial, or fully-vectorial BPM for 3D structures [15, 18-20].

Indeed, the main problem of these above-mentioned BPMs is the paraxiality and unidirectionality of considered wave propagation. Paraxiality can be removed by using Padé approximants or by employing the Method of Lines (MoL) [14]. Unidirectionality can be removed by using bidirectional formulation of BPM (BiBPM) [14]. It is very important to consider correctly the influence of evanescent waves for application of BiBPM in modelling the near-field optical storage system. Next, we will show the way to develop a BPM satisfying these requirements.

\section{2D nonparaxial BPM}

For simplicity, 2D geometry and propagation of TE polarized beam are considered. The beam propagates along $z$ axis, $x$ axis is transverse coordinate, the wave electric vector has only $E_{y}$ component oriented along $y$ axis of the coordinate system. The propagation of this light beam is described by the Helmholtz wave equation

$$
\frac{\partial^{2} E_{y}}{\partial z^{2}}+\frac{\partial^{2} E_{y}}{\partial x^{2}}+k_{0}^{2} n_{\mathrm{ef}}^{2} E_{y}=0
$$

where $n_{\text {ef }}^{2}(x, z)=n^{2}(x, z)+G(x, z) / E_{y}$ is the effective refraction index, $n(x, z)$ is the linear index distribution, $G(x, z)=\chi^{(3)} E_{y}^{3}$ is Kerr nonlinearity, $k_{0}=2 \pi / \lambda$ is a wavenumber, $\lambda$ is a wavelength. Next, we will extract the reference index $n_{0}$, i.e. we introduce envelope $E(z, x)$ of propagating wave amplitude $E_{y}=E \exp \left(-\mathrm{i} k_{0} n_{0} z\right)$, where $\mathrm{i}=\sqrt{-1}$, and Eq. (1) can be rewritten as follows for wave envelopes:

$$
-\frac{1}{k_{0}^{2} n_{0}^{2}} \frac{\partial^{2} E}{\partial z^{2}}+\frac{2 \mathrm{i}}{k_{0} n_{0}} \frac{\partial E}{\partial z}=\bar{P} E
$$

where

$$
\bar{P}=\frac{1}{k_{0}^{2} n_{0}^{2}}\left[\frac{\partial^{2}}{\partial x^{2}}+k_{0}^{2}\left(n^{2}-n_{0}^{2}\right)+k_{0}^{2} \frac{G}{E}\right]
$$

is the nonlinear wave propagation operator.

If we assume that

$$
\left|\frac{\partial^{2} E}{\partial z^{2}}\right| \ll\left|2 \mathrm{i} k_{0} n_{0} \frac{\partial E}{\partial z}\right|,
$$

then the second $z$ derivative in Eq. (2) can be omitted and the paraxial wave propagation equation is obtained. Nonparaxial approach takes into account this $z$ derivative describing longitudinal diffraction. One of the best and stable methods to evaluate this second derivative is Padé approximants for the propagation operator $[14,15]$. The second $z$ derivative in the Helmholtz equation (2) means not only nonparaxial propagation but also two-way wave propagation in the forward and backward directions. Therefore, we can split Eq. (2) into two one-way equations for waves propagating forward $\left(E_{+}\right)$and backwards $\left(E_{-}\right)$,

$$
\frac{\partial E_{ \pm}}{\partial z}=-\mathrm{i} k_{0} n_{0}[-1 \mu \sqrt{1+\bar{P}}] E_{ \pm} .
$$

Now, the nonparaxiality of wave propagation is described by the square root operator. To take into account exactly this square root operator, for each propagation step we would need to solve the eigenvalue problem [21]. Of course, this is a very computationally expensive and slow approach, realized in the MoL method [22]. It is possible to expand the square root in Eq. (4) by Taylor series. But this way is also not efficient because it leads to complicated multi-point finitedifference schemes. Today, the approach based on using Padé approximants [23] to evaluate the square root operator in Eq. (4) is recognized as the most efficient way to solve the nonparaxiality problem. The usage of Padé approximants deals with replacing square root in Eq. (4) by a ratio of two polynomials $N$ and $D$ in propagation operator $\bar{P}$ :

$$
\frac{\partial E_{+}}{\partial z}=-\mathrm{i} k_{0} n_{0} \frac{N}{D} E_{+} .
$$


Table 1. Supported beam spreading angle $\theta$ by different orders of Padé approximants.

\begin{tabular}{cccccc}
\hline Padé order & $(1,0)$ & $(1,1)$ & $(2,2)$ & $(3,3)$ & $(4,4)$ \\
\hline$\theta$ & $15^{\circ}$ & $30^{\circ}$ & $48^{\circ}$ & $60^{\circ}$ & $67^{\circ}$ \\
\hline
\end{tabular}

There are a few of ways to build these polynomials. One way is to derive from Eq. (2) this iterative formula

$$
\frac{\partial E_{+}}{\left.\partial z\right|_{M}}=-\mathrm{i} k_{0} n_{0} \frac{\frac{\bar{P}}{2} E_{+}}{1+\frac{\mathrm{i}}{2 k_{0} n_{0}} \frac{\partial}{\left.\partial z\right|_{M-1}}},
$$

where $M$ is iteration order, and get expressions for various orders $n$ of Padé $(n, n)$ polynomials in the ratios of numerators $N$ and denominators $D$. For paraxial approach we have Padé $(1,0)$ approximant

$$
\frac{N}{D}=\frac{\bar{P}}{2}
$$

then the first nonparaxial approach with Padé $(1,1)$ approximant

$$
\frac{N}{D}=\frac{\frac{\bar{P}}{2}}{1+\frac{\bar{P}}{4}}
$$

then the next Padé $(2,2)$ approximant

$$
\frac{N}{D}=\frac{\frac{\bar{P}}{2}+\frac{\bar{P}^{2}}{4}}{1+\frac{3 \bar{P}}{4}+\frac{\bar{P}^{2}}{16}},
$$

etc.

Table 1 summarizes validity ranges of different order Padé approximants for propagation of beams with a spreading angle $\theta[24,25]$. The results of nonparaxial beam propagation are presented in the limit of $1 \%$ errors.

Further, for development of BiBPM, it is convenient to transform Eqs. (4) for wave envelopes to a more symmetric form for fast wave amplitudes without extraction of reference index:

$$
\begin{aligned}
& \frac{\partial E_{y+}}{\partial z}=-\mathrm{i} k_{0} n_{0} \sqrt{1+\bar{P}} E_{y+}, \\
& \frac{\partial E_{y-}}{\partial z}=-\mathrm{i} k_{0} n_{0} \sqrt{1+\bar{P}} E_{y-},
\end{aligned}
$$

and Padé $(n, n)$ polynomials are transformed into the form useful for developing multistep algorithms:

$$
\sqrt{1+\bar{P}}=\frac{\tilde{N}}{\tilde{D}}=C_{n} \prod_{i=1}^{n} \frac{\bar{P}-c_{i}}{\bar{P}-d_{i}} .
$$

In Eq. (9), the Padé $(n, n)$ polynomial is presented as a product of $n$ Padé $(1,1)$ approximants. This allows us to use split-step algorithm for Padé $(n, n)$ polynomials and to reduce the whole problem to a multiple application of Padé $(1,1)$ approximants [26] preserving the tridiagonal structure of numerical schemes. It is a great benefit in comparison with the method of using expansions of the square root operator by Taylor series or MoL approach.

\section{Evanescent wave description by complex Padé approximants}

In order to study near-field optical recording it is important to properly describe the evanescent field that dominates in the near-field optics. The main problem is that the usual BPM does not support evanescent waves at all. In the FFT-BPM, evanescent spectrum of field is filtered out due to stability problems [16]. In the FDBPM, evanescent waves are considered as ones propagating without any damping [27] in the wave propagation ( $z$ axis) direction. Introduction of Padé approximants in the FD-BPM for wide-angle wave propagation also does not resolve this problem. Padé approximants in Eq. (9) have real roots, i.e. real poles in the evanescent wave region. Therefore these expressions are singular and invoke numerical instabilities for situations with large contribution of evanescent field to the total wave field, which happens for near-field optics. This problem of stability can be fixed by introducing the complex Padé approximants using some algorithm to convert real roots of Padé polynomials to complex ones.

One way to introduce complex Padé approximants was suggested by Vassalo [28] for the fourth root operator $\sqrt[4]{1+\bar{P}}$, which we will apply for the square root operator in Eq. (9). The idea is to use the transformation of the propagation operator, $\bar{P}=\mathrm{i} s+(1+\mathrm{i} s) \bar{\sigma}$, that performs shifting of zeros and poles on the complex plane. Using this substitution, we can derive new expression of Eq. (9)

$$
\sqrt{1+\bar{P}}=C_{n} \sqrt{1+\mathrm{i} s} \prod_{i=1}^{n} \frac{\bar{P}-\tilde{c}_{i}}{\bar{P}-\tilde{d}_{i}}
$$




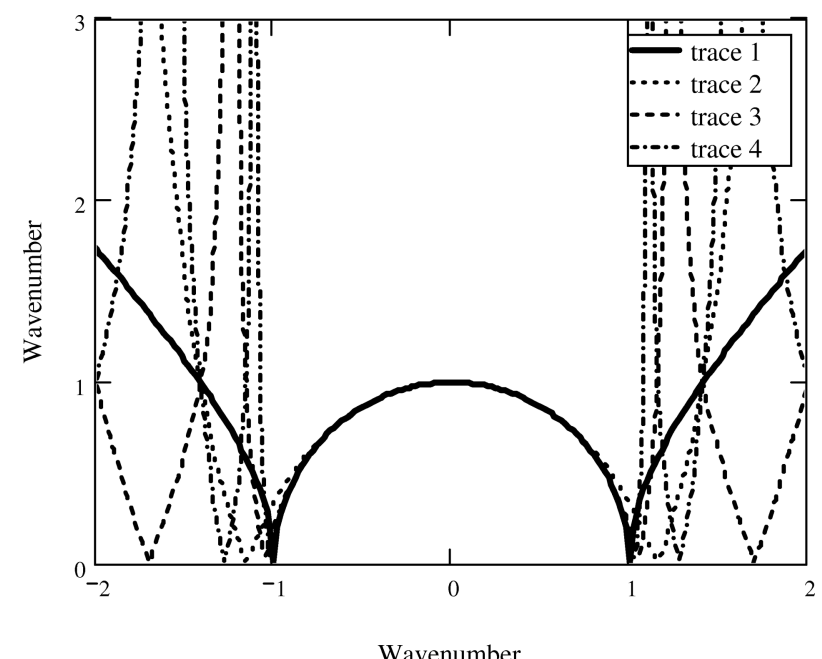

(a)

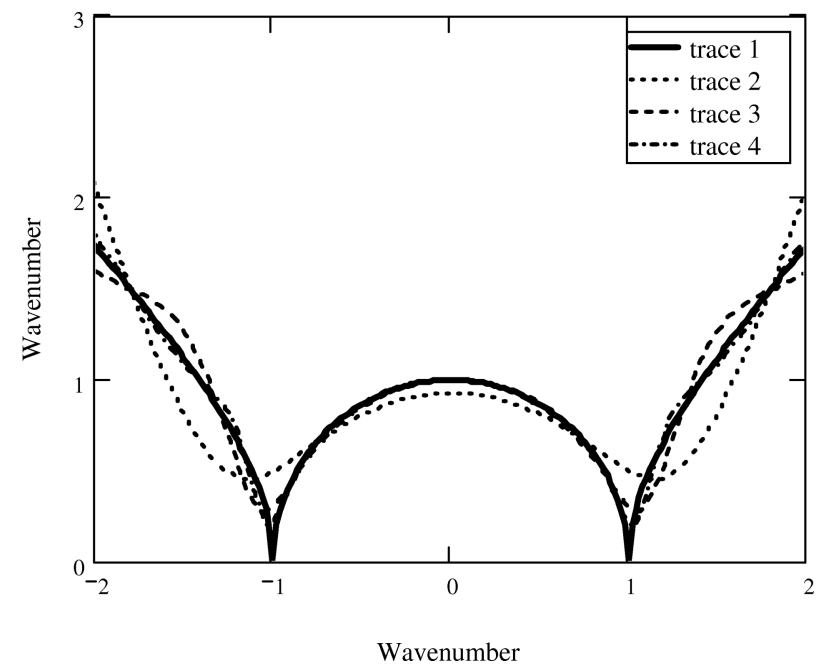

(c)

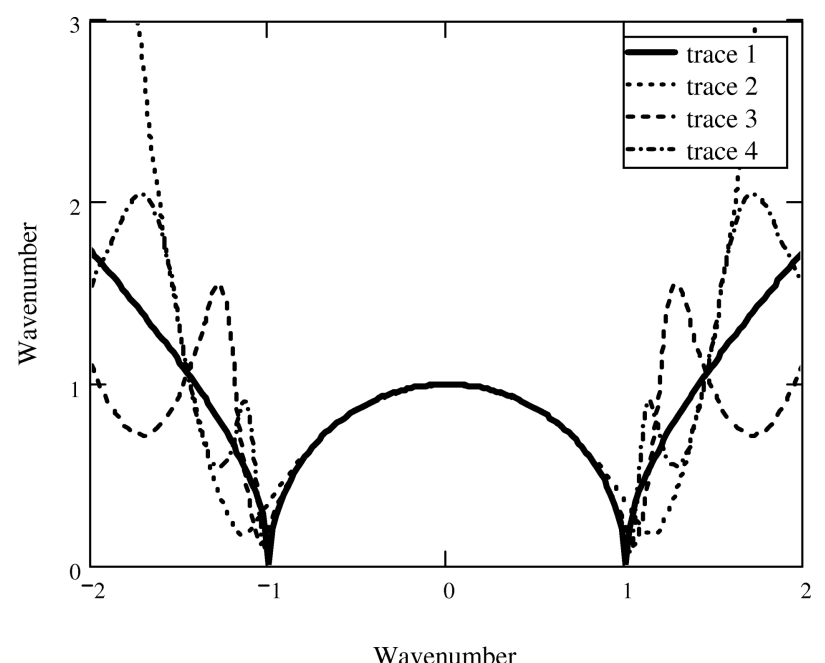

(b)

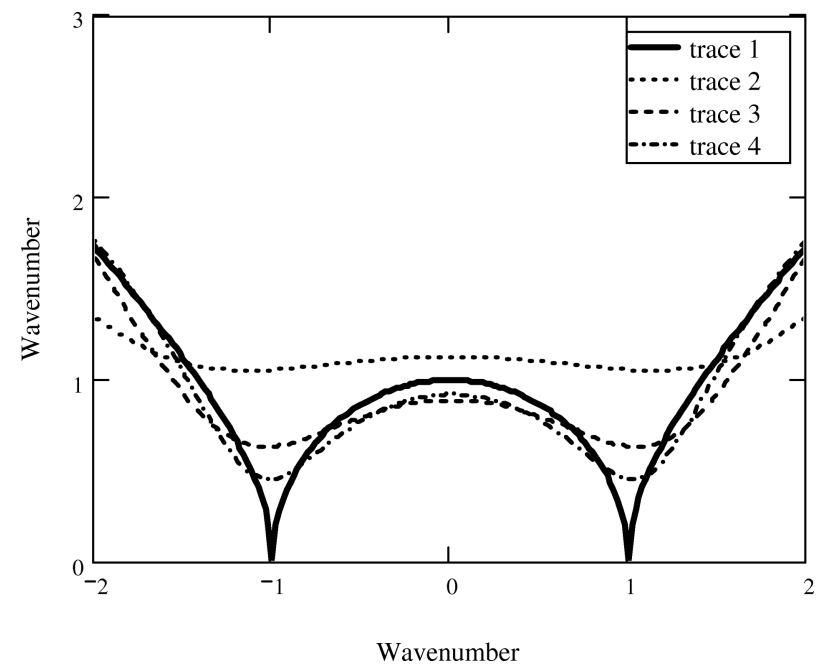

(d)

Fig. 1. Approximation of Ewald sphere by complex Padé approximants created by the pole-zero shifting method versus shifting parameter $s$, (a) $s=0$, (b) $s=0.5$, (c) $s=2.0$, (d) $s=10$, where trace 1 is exact Ewald sphere, trace 2 is complex Padé (1, 1), trace 3 is complex Padé $(2,2)$, trace 4 is complex Padé $(3,3)$ approximant.

where $s$ is the shifting parameter and the new roots of complex Padé polynomials are

$$
\tilde{c}_{i}=\mathrm{i} s+c_{i}(1+\mathrm{i} s), \quad \tilde{d}_{i}=\mathrm{i} s+d_{i}(1+\mathrm{i} s) .
$$

In Fig. 1 we show approximation of modulus of the square root operator (10) for the case of plane wave propagation in the free space. In this case, the square root operator represents Ewald sphere [29], which displays behaviour of wavenumber in longitudinal propagation direction versus wavenumber in transverse direction in normalized units. Trace 1 in Fig. 1(a-d) shows exact solution for Ewald sphere. Propagating waves described by a semicircle in the $K$-space for isotropic medium appear in the region $[-1,1]$. The regions $[-\infty,-1]$ and $[1,+\infty]$ contain evanescent waves. For the case of real Padé roots $(s=0$, Fig. 1(a)) we can see the poles in the evanescent regions and the Padé approximants do not describe these waves. By increasing the shifting parameter $s$ (Figs. 1(b,c)) the smoothing of poles occurs and sufficiently exact "global" approximation of the total Ewald sphere by Padé $(3,3)$ approximant (Fig. 1(c)) is built. A further increase of the shifting parameter $(s=10$, Fig. 1(d)) leads to greater errors for approximation of propagating waves.

Another way to introduce complex Padé approximants was suggested by Brooke [30]. It is the method of rotation of square root branch-cut in the complex Riemann plane, which is carried out by using the transformation of the square root operator $\sqrt{1+\bar{P}}=$ 
$\exp (\mathrm{i} \alpha / 2) \sqrt{1+\bar{\sigma}_{1}}, \bar{\sigma}_{1}=\exp (-\mathrm{i} \alpha)(1+\bar{P})-1$, and leads to the following new expression for Eq. (9):

$$
\sqrt{1+\bar{P}}=\exp (\mathrm{i} \alpha / 2) C_{n} \prod_{i=1}^{n} \frac{\bar{P}-\hat{c}_{i}}{\bar{P}-\hat{d}_{i}},
$$

where $\alpha$ is angle of branch-cut rotation and the new complex Padé roots are

$\hat{c}_{i}=\exp (\mathrm{i} \alpha)\left(1+c_{i}\right)-1, \quad \hat{d}_{i}=\exp (\mathrm{i} \alpha)\left(1+d_{i}\right)-1$.

For the case of $\alpha=0^{\circ}$, we again have real Padé roots and the same result as shown in the Fig. 1(a). By increasing the rotation angle (Figs. 2(a,b)) up to $\alpha=90^{\circ}$, we can see smoothing of poles in the evanescent wave region and almost perfect approximation of the "global" Ewald sphere by complex Padé $(3,3)$ ap-

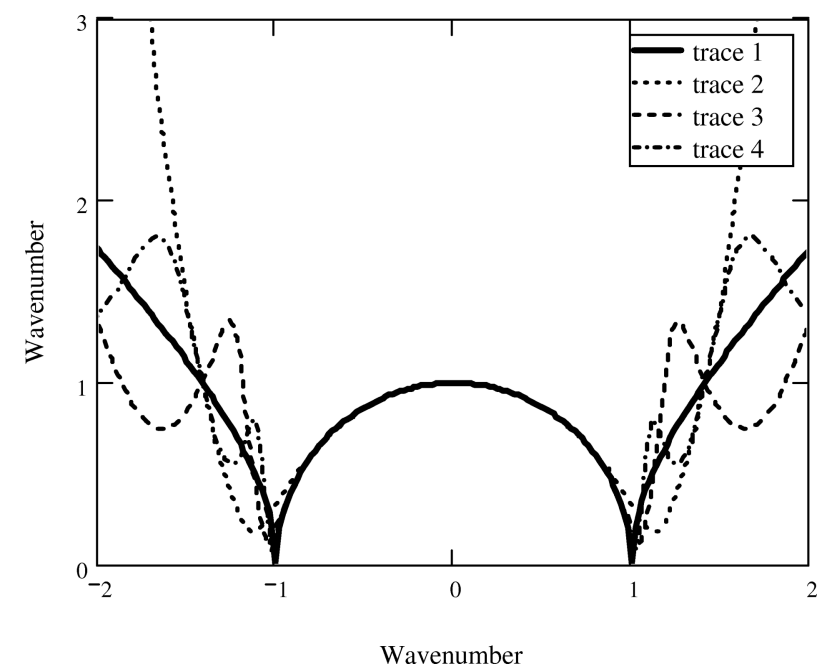

(a)

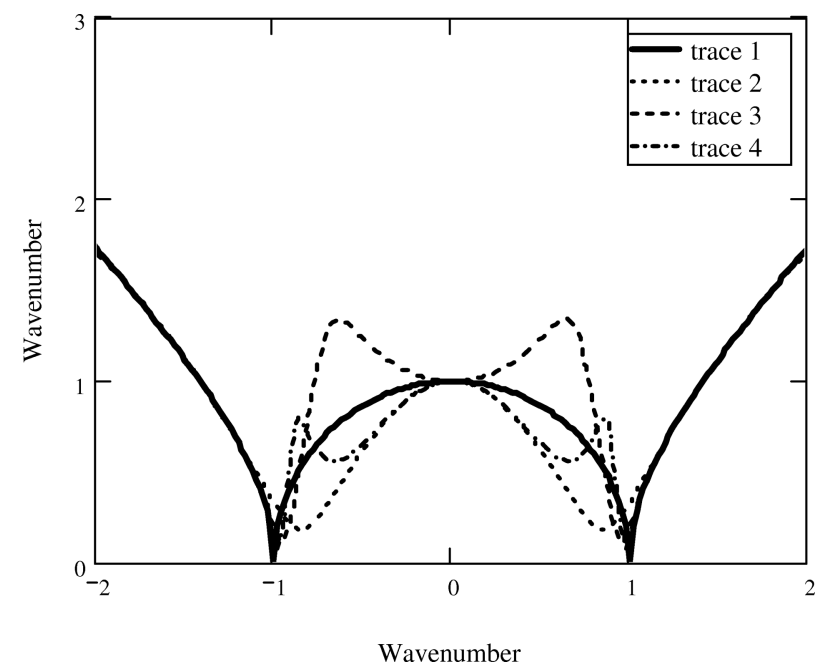

(c) proximant. Further increasing of the rotation angle invokes the appearance of poles in the region of propagating waves (Fig. 2(c)) and complete breakdown of propagating wave description by complex Padé approximants (Fig. 2(d)).

It is necessary to note that, as shown in [27], the use of the branch-cut rotation method is equivalent to the use of complex reference index. This guess of wave with complex carrier frequency can be not applicable to some problems of interest as it produces a strong power loss. We can also see some differences in the behaviour of the best approximation to Ewald sphere (Figs. 1(c) and 2(b)) by using pole-zero shifting and branch-cut rotation methods. For lower order Padé approximants, the shifting method shows larger errors in the propagating wave region, which then disappear by

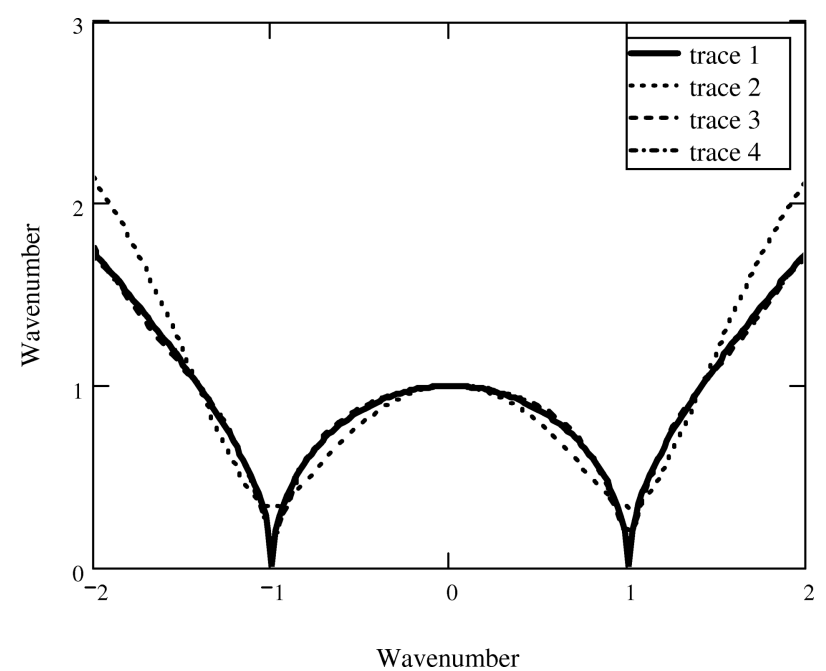

(b)

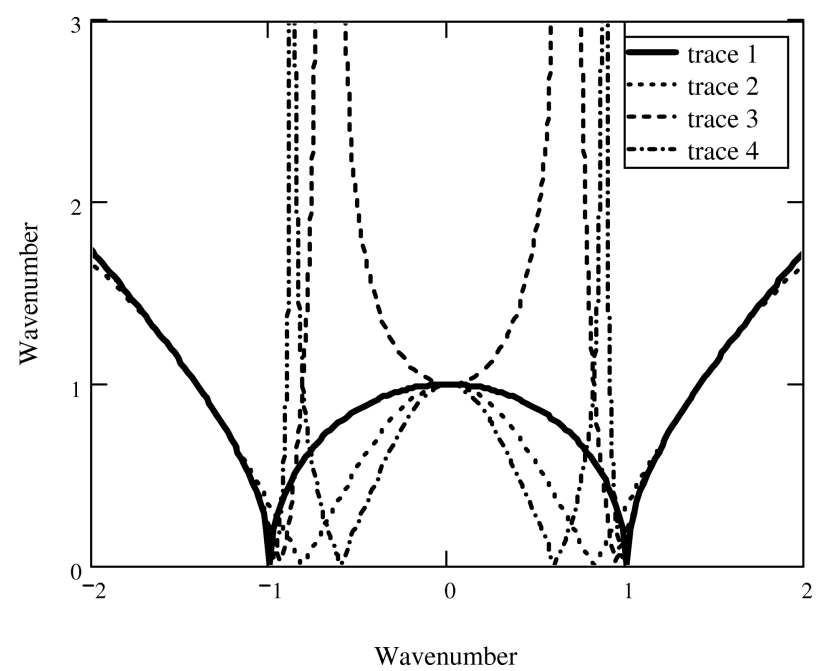

(d)

Fig. 2. Approximation of Ewald sphere by complex Padé approximants created by the branch-cut rotation method versus rotation angle $\alpha$, (a) $\alpha=30^{\circ}$, (b) $\alpha=90^{\circ}$, (c) $\alpha=150^{\circ}$, (d) $\alpha=180^{\circ}$, where trace 1 is exact Ewald sphere, trace 2 is complex Padé $(1,1)$, trace 3 is complex Padé $(2,2)$, trace 4 is complex Padé $(3,3)$ approximant. 


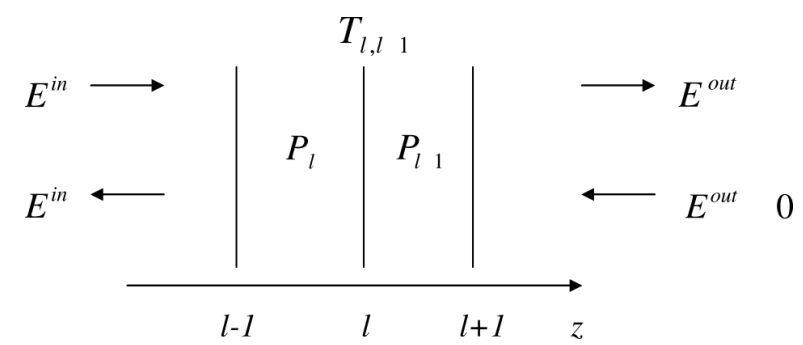

Fig. 3. Bidirectional propagation in multiple interface structure.

increasing the approximant order. For the case of the rotation method at the lower order Padé approximants, we encounter larger errors in off-axis region of propagating waves, which again then disappear by increasing the approximant order.

\section{2D bidirectional BPM}

Next, we will show how it is possible in the BPM algorithm to take into consideration the beam reflections. As an example, the TE polarized beam propagation through the layered system shown in Fig. 3 is considered. This reflective structure consists of layers $l$ with distinct refraction indices $n_{l}(x, z)$. The beam $E_{+}^{\text {in }}(x, z)$ impinges on this structure and produces reflected $E_{-}^{\text {in }}(x, z)$ and transmitted $E_{+}^{\text {out }}(x, z)$ beams. For convenience, the following notation for the square root operator in the layer $l$ is introduced:

$$
Q_{l}=k_{0} n_{0} \sqrt{1+\bar{P}^{(l)}}=k_{0} n_{0} \frac{\widetilde{N}^{(l)}}{\widetilde{D}^{(l)}},
$$

and we rewrite one-way symmetric Eqs. $(7,8)$ in the form

$$
\begin{aligned}
& \frac{\partial E_{y+}^{(l)}}{\partial z}=-\mathrm{i} Q_{l} E_{y+}^{(l)}, \\
& \frac{\partial E_{y-}^{(l)}}{\partial z}=-\mathrm{i} Q_{l} E_{y-}^{(l)} .
\end{aligned}
$$

The formal solution of Eqs. $(15,16)$ in the operator form in the layer $l$ is

$$
\begin{aligned}
E_{y}^{(l)} & =E_{y+}^{(l)}+E_{y-}^{(l)} \\
& =\exp \left(-\mathrm{i} Q_{1} z_{1}\right) A_{1}+\exp \left(\mathrm{i} Q_{1} z_{1}\right) B_{1} .
\end{aligned}
$$

To describe the beam transmission from layer $l$ to $l+1$ we apply internal boundary conditions for full electric and magnetic light wave fields

$$
\begin{aligned}
& E_{y}^{(l)}=E_{y}^{(l+1)}, \\
& H_{x}^{(l)}=H_{x}^{(l+1)} .
\end{aligned}
$$

After substitution of (17) into Eqs. $(18,19)$ it is possible to derive transmission operator $T_{l, l+1}$ from layer $l$ to layer $l+1$

$$
T_{l, l+1}=\frac{1}{2}\left|\begin{array}{ll}
1+Q_{l} / Q_{l+1} & 1-Q_{l} / Q_{l+1} \\
1-Q_{l} / Q_{l+1} & 1+Q_{l} / Q_{l+1}
\end{array}\right|
$$

and a new propagation operator

$$
P_{l}=\left|\begin{array}{cc}
P_{l}^{+} & 0 \\
0 & P_{l}^{-}
\end{array}\right|=\left|\begin{array}{cc}
\exp \left(-\mathrm{i} Q_{l} z_{l}\right) & 0 \\
0 & \exp \left(\mathrm{i} Q_{l} z_{l}\right)
\end{array}\right| .
$$

The total algorithm of beam bidirectional propagation in the operator form can be written as follows:

$$
\left|\begin{array}{l}
E_{+}^{\text {out }} \\
E_{-}^{\text {out }}
\end{array}\right|=M\left|\begin{array}{l}
E_{+}^{\text {in }} \\
E_{-}^{\text {in }}
\end{array}\right|
$$

where $M$ is some product of propagation and transmission operators,

$$
M=\left|\begin{array}{ll}
g_{11} & g_{12} \\
g_{21} & g_{22}
\end{array}\right|=T_{n, n+1} P_{n} \ldots P_{2} T_{1,2} P_{1} .
$$

For beam propagation in the linear layered structure, Eqs. (22) can be solved by direct and noniterative way [31] or by applying some iterative algorithm [32]. For example, using the direct solution of Eqs. (22), after some manipulations with operators, we obtain $E_{-}^{\text {in }}=-g_{22}^{-1} g_{21} E_{+}^{\text {in }}$ for the reflected beam. We get the formal solution in the operator form $E_{-}^{\text {out }}=\left(g_{11}-g_{12} g_{22}^{-1} g_{21}\right) E_{+}^{\text {in }}$ for the transmitted beam as well. For the beam propagation through the nonlinear layered structure only iterative methods are suitable [33]. In the case of plane wave propagation in the structure with plane layers Eqs. $(22,23)$ turn into the wellknown transfer matrix method (TMM) [29], which is widely used in simulation of optical coatings and interference filters. In our case of BiBPM, the matrix Eqs. $(22,23)$ include differential operators with partial derivatives. Therefore, it is a quantitatively new method. We again use complex Padé approximants for evaluation of square root operators in the numerical schemes and this BiBPM algorithm has only formal similarity to TMM. For long distance beam propagation in the layered structure of high contrast of refraction index the scattering matrix formulation of BiBPM is more preferable $[34,35]$. This formulation is more complicated in numerical implementation but it is more stable for the cases of structures of high index contrast.

As a numerical example of application of bidirectional and nonparaxial BPM with complex Padé approximants, we consider modelling of reflection of narrow, nonparaxial Gaussian beam from a polymer slab on a high index $\mathrm{GaP}$ substrate. The propagation of 


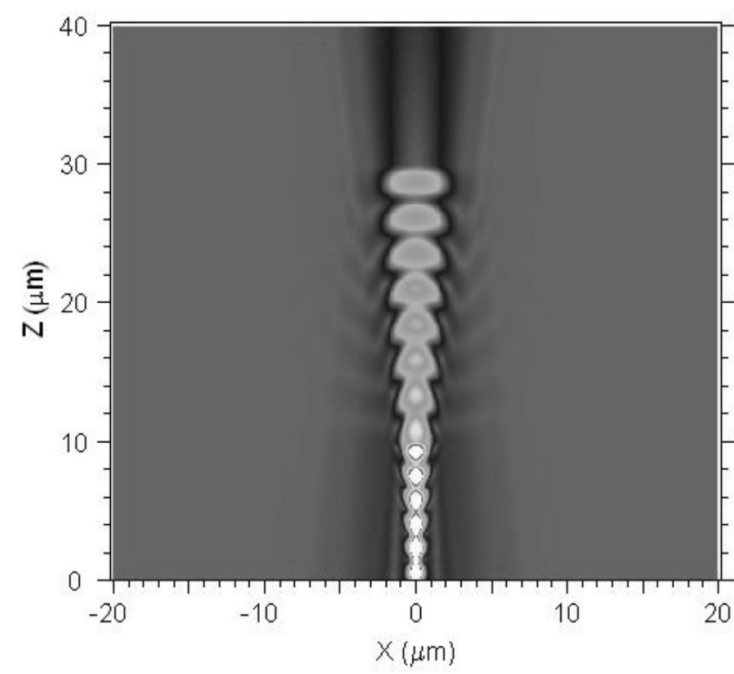

(a)

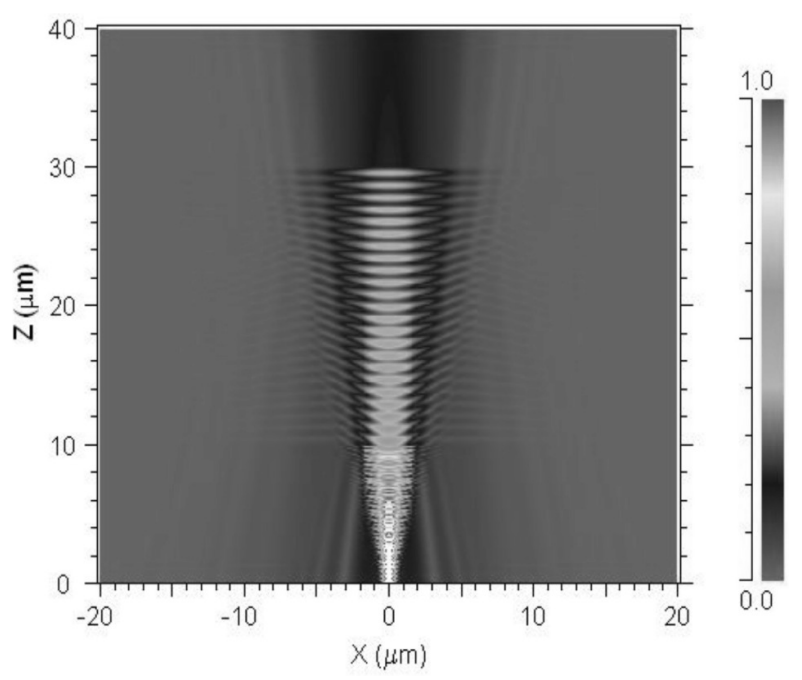

(b)

Fig. 4. Modelling of nonparaxial Gaussian beam reflection from polymer slab on GaP substrate, (a) using Padé $(1,0)$ approximants (paraxial approach) and (b) using Padé $(3,3)$ approximants for beam propagation and reflection operators.

Gaussian beam with $0.8 \mu \mathrm{m}$ full width at the waist and $0.4 \mu \mathrm{m}$ wavelength is studied. The polymer slab had $20 \mu \mathrm{m}$ thickness and refraction index 1.6. GaP substrate had refraction index 3.2. The results of modelling of this wide-angle beam reflection are presented in Fig. 4. The complex Padé approximants obtained by pole-zero shifting method are used with shifting parameter $s=0.6$. Figure 4(a) presents results of paraxial modelling using Padé $(1,0)$ approximants for beam propagation and reflection from interfaces. Figure 4(b) shows results of the same beam nonparaxial modelling using Padé $(3,3)$ approximants for evaluation of beam propagation and reflection operators. We can clearly see that paraxial approach does not simulate correctly the narrow beam propagation and reflection, and the improvement of beam propagation is achieved by increasing the order of Padé approximants for evaluation of propagation and reflection operators.

\section{Conclusion}

The algorithm of the nonparaxial and bidirectional beam propagation method suitable for modelling nearfield optical data storage systems is suggested. The formulation of BiBPM for 2D geometry and TE polarization of incident light beams is derived. Complex Padé approximants are introduced for correct approximation of evanescent field in the wave propagation direction. Pole-zero shifting and branch-cut rotation methods of building complex Padé approximants are studied and compared. Bidirectional generalization of the BPM algorithm is obtained. The suggested method turns into a well-known transfer matrix method for the case of study of plane wave propagation in a layered structure with plane layers. The BiBPM algorithm based on nonparaxial solution of the Helmholtz wave equation should be faster and request less computer resources then the traditional FDTD method used for simulation of near-field and high NA optical storage systems.

\section{Acknowledgement}

This research was partially supported by the Lithuanian State Science and Studies Foundation grant COST P8, Reg. No. V-04033.

\section{References}

[1] E. Betzing, J.K. Trautman, R. Wolfe, E.M. Gyorgy, P.L. Finn, M.H. Kryder, and C.-H. Chang, Near-field magneto-optics and high density data storage, Appl. Phys. Lett. 61, 142-144 (1992).

[2] K. Kato, S. Ichihara, M. Oumi, H. Maeda, T. Niwa, T. Mitsuoka, K. Nakajima, T. Ohkubo, and K. Itao, Signal readout using small near-field optical head with horizontal light introduction through optical fiber, Jpn. J. Appl. Phys. 42, 5102-5106 (2003).

[3] K. Goto, Y.-J. Kim, S. Mitrugi, K. Kurihara, and T. Horibe, Microoptical two-dimensional devices for the optical memory head of ultrahigh data transfer rate and density system using a vertical cavity surface emitting laser (VCSEL) array, Jpn. J. Appl. Phys. 41, 48354840 (2002).

[4] J. Hashizume, S. Shinada, and F. Koyama, Near-field optical probing using a microaperture GaInAs/GaAs 
surface emitting laser, Jpn. J. Appl. Phys. 41, L700L702 (2002).

[5] B.D. Terris, H.J. Mamin, and D. Rugar, Near-field optical data storage. Appl. Phys. Lett. 68, 141-143 (1996).

[6] T.D. Milster, J.S. Jo, and K. Hirota, Roles of propagating and evanescent waves in solid immersion lens systems, Appl. Opt. 38, 5046-5067 (1999).

[7] J. Tominaga, T. Nakano, and N. Atoda, An approach for recording and readout beyond the diffraction limit with an Sb thin film, Appl. Phys. Lett. 73, 2078-2080 (1998).

[8] T. Nakano, Y. Yamakawa, J. Tominaga, and N. Atoda, Near-field optical simulation of super-resolution nearfield structure disks, Jpn. J. Appl. Phys. 40, 1531-1535 (2001).

[9] W.-C. Lin and D.P. Tsai, Nonlinear near-field optical effects of the $\mathrm{AgO}_{x}$-type super-resolution near-field structure, Jpn. J. Appl. Phys. 42, 1031-1032 (2003).

[10] J. Guerra, D. Vezenov, P. Sullivan, W. Haimberger, and L. Thulin, Near-field optical recording without lowflying heads: Integral near-field optical (INFO) media, Jpn. J. Appl. Phys. 41, 1866-1875 (2002).

[11] A. Taflove and S.C. Hagness, Computational Electrodynamics: The Finite-Difference Time-Domain Method, 2nd ed. (Artech House, Boston, 2000).

[12] K.S. Yee, Numerical solution of initial boundary value problems involving Maxwell's equations in isotropic media, IEEE Trans. Antennas Propag. 14, 302-307 (1966).

[13] H.J.W.M. Hoekstra, On beam propagation methods for modeling in integrated optics, Opt. Quantum Electron. 29, 157-171, 1997.

[14] R. Scarmozzino, A. Gopinath, R. Pregla, and S. Helfert, Numerical techniques for modeling guidedwave photonic devices, IEEE J. Select. Top. Quantum Electron. 6, 150-162 (2000).

[15] J. Yamauchi, Propagating Beam Analysis of Optical Waveguides (Research Studies Press, Baldock, 2003).

[16] M.D. Feit and J.A. Fleck, Light propagation in gradedindex optical fibers, Appl. Opt. 17, 3990-3998 (1978).

[17] Y. Chung and N. Dagli, An assessment of finite difference beam propagation method, IEEE J. Quantum Electron. 26, 1335-1339 (1990).

[18] W.P. Huang, Simulation of three-dimensional optical waveguides by full-vector beam propagation method, IEEE J. Quantum Electron. 29, 2639-2649 (1993).

[19] E.E. Kriezis and A.G. Papagiannakis, A threedimensional full vectorial beam propagation method for $z$-dependent structures, IEEE J. Quantum Electron. 33, 883-890 (1997).

[20] F. Fogli, G. Bellanca, P. Bassi, I. Madden, and W. Johnstone, Highly efficient full-vectorial 3-D BPM modeling of fiber to planar waveguide coupler, J. Lightwave Tech. 17, 136-143 (1999).
[21] Y.-P. Chiou and H.-C. Chang, Analysis of optical waveguide discontinuities using the Padé approximants, IEEE Ph. Tech. Lett. 9, 964-966 (1997).

[22] S.F. Helfert and R. Pregla, The method of lines: A versatile tool for the analysis of waveguide structures, Electromagnetics 22, 615-637 (2002).

[23] G.R. Hadley, Wide-angle beam propagation using Padé approximant operators, Opt. Lett. 17, 1426-1428 (1992).

[24] F. Ma, C.L. Xu, and W.P. Huang, Wide-angle full vectorial beam propagation method, IEE Proc. Optoelectron. 143, 139-143 (1996).

[25] R. Petruškevičius, G. Bellanca, and P. Bassi, BPM modeling of three-wave interaction in periodically poled second-order nonlinear materials, Lithuanian J. Phys. 38, 168-176 (1998).

[26] G.R. Hadley, Multistep method for wide-angle beam propagation, Opt. Lett. 17, 1743-1745 (1992).

[27] H. Rao, M.J. Stell, R. Scarmozzino, and R.M. Osgood, Complex propagators for evanescent waves in bidirectional beam propagation method, J. Lightwave Tech. 18, 1155-1160 (2000).

[28] C. Vassallo, Limitations of the wide-angle beam propagation method in nonuniform systems, J. Opt. Soc. Am. A 13, 761-770 (1996).

[29] M. Born and E. Wolf, Principles of Optics (Pergamon Press, Oxford, 1980).

[30] F.A. Milinazzo, C.A. Zala, and G.H. Brooke, Rational square-root approximations for parabolic equation algorithms, J. Acoust. Soc. Am. 101, 760-766 (1997).

[31] H. El-Refaei, D. Yevick, and I. Betty, Stable and noniterative bidirectional beam propagation method, IEEE Ph. Tech. Lett. 12, 389-391 (2000).

[32] H. Rao, R. Scarmozzino, and R. Osgood, A bidirectional beam propagation method for multiple dielectric interfaces, IEEE Ph. Tech. Lett. 11, 830-832 (1999).

[33] A. Locatelli, F.-M. Pigozzo, F. Baranio, D. Modotto, A.-D. Capobianco, and C. De Angelis, Bidirectional beam propagation method for second-harmonic generation in engineered multilayered waveguides, Opt. Quantum. Electron. 35, 429-452 (2003).

[34] P.L. Ho and Y.Y. Lu, A stable bidirectional propagation method based on scattering operators, IEEE Ph. Tech. Lett. 13, 1316-1318 (2001).

[35] A. Locatelli, D. Modotto, C. De Angelis, F.-M. Pigozzo, and A.-D. Capobianco, Nonlinear bidirectional beam propagation method based on scattering operators for periodic microstructured waveguides, J. Opt. Soc. Am. B 20, 1724-1731 (2003). 


\title{
KOMPLEKSINIAI PADÉ ARTINIAI DVIEJU KRYPČIŲ IR NEGRETAAŠIAME PLUOŠTO SKLIDIMO METODE
}

\author{
R. Petruškevičius
}

Fizikos institutas, Vilnius, Lietuva

\section{Santrauka}

Negretaašis ir dviejų krypčių pluošto sklidimo metodas, tinkantis modeliuoti artimo lauko ir didelès skaitmeninès apertūros optinio duomenu užrašymo sistemas, pasiūlytas $2 \mathrm{D}$ geometrijai ir TE krentančio pluošto poliarizacijai. Kompleksiniai Padé artiniai ịvesti korektiškam nespindulinio lauko aprašymui artimo lauko optikoje. Nagrinèti ir palyginti polių-nuliu postūmio ir šaknies pjūvio sukimo kompleksinių Padé artinių suformavimo metodai. 